\title{
Temporally resolved studies of thermal effects in high power ZGP OPO pumped by high-repetition Ho:LLF MOPA system
}

\section{Marcin Piotrowski ${ }^{1}$, Manuel-Alessandro Medina ${ }^{1,2}$, Martin Schellhorn ${ }^{1}$, Anne Hildenbrand-Dhollande ${ }^{1}$ \\ 1. French-German Research Institute of Saint-Louis (ISL), 5 rue du Général Cassagnou, 68301 Saint-Louis, France \\ 2. Aix Marseille Univ., CNRS, Centrale Marseille, Institut Fresnel, Marseille, France}

Tunable high power laser sources covering mid-IR region $(3-5 \mu \mathrm{m})$ find numerous applications in remote sensing, free space communication or defence countermeasures. Besides high power, such applications require a broad tuning range and a good beam quality. The optical parametric oscillation (OPO) and amplification (OPA) with non-linear zinc germanium phosphide (ZGP) crystals is a main approach to deliver such laser sources [1]. To date, there are only few reports of high average power from ZGP OPOs, often with a compromised beam quality and stability [2-4]. Here, we experimentally investigate methods to enhance a good beam quality, focusing particularly on the aspect of different cavity geometries, lengths, and the influence of a pump repetition rate.

We employed a high-efficient resonantly pumped Q-switched Ho:LLF MOPA $10 \mathrm{kHz}$ system as a pump source for the ZGP OPO. The Ho:LLF pump delivers $68.7 \mathrm{~W}$ at $2065 \mathrm{~nm}$ in TEM To $_{0}$ operation at the repetition rate of 10 $\mathrm{kHz}$ with an optical-to-optical efficiency of $61.5 \%$ [5].

Our single stage ZGP OPO generates $38 \mathrm{~W}$ of total power in a linear cavity and above $20 \mathrm{~W}$ in non-planar ring cavities RISTRA and FIRE [6-8] with $\mathrm{M}^{2}<1.5$. Fig. 1 contains the example results for the $130 \mathrm{~mm}$ long RISTRA cavity. The left graph shows how the beam quality $\left(\mathrm{M}^{2}\right)$ changes with pump pulse energy at the fixed repetition rate of $10 \mathrm{kHz}$. The right graph shows the thermal effects build-up process. To capture the $\mathrm{M}^{2}$ factor as a time function we collected beam images at different positions along the beam at $50 \mathrm{~Hz}$ frame rate $(20 \mathrm{~ms}$ time resolution) using pyroelectric array sensor and reconstructed the beam profile in post-processing.
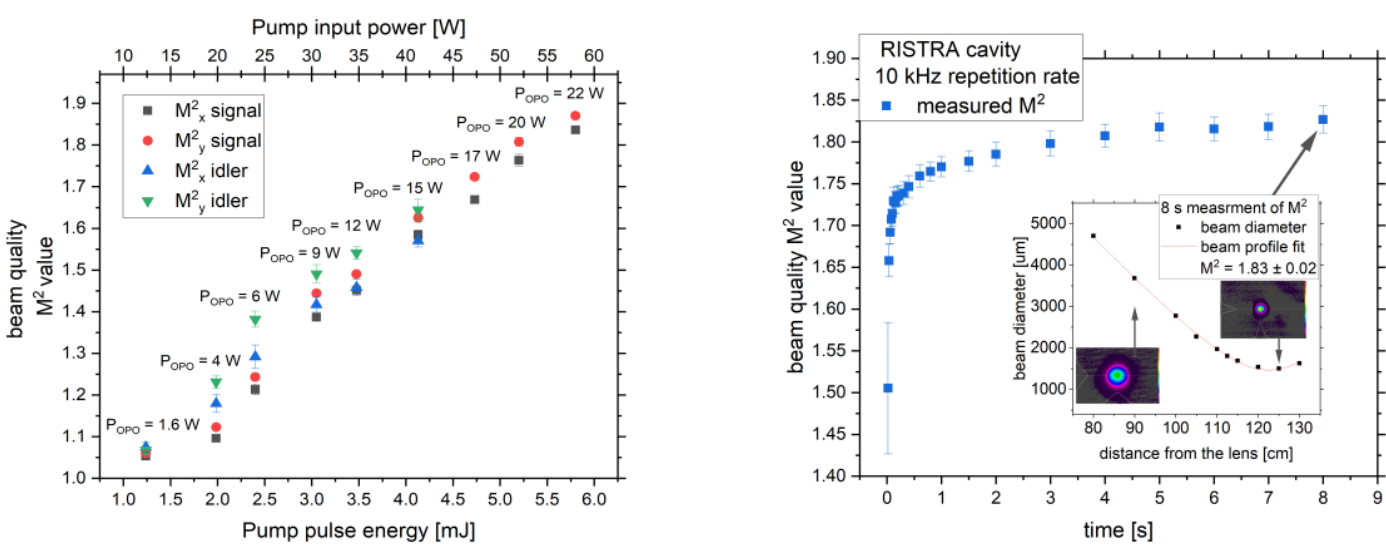

Fig. 1 Left: The OPO output beam $\mathrm{M}^{2}$ as a function of pump pulse energy at the $10 \mathrm{kHz}$ repetition rate. The labels indicate the total (signal + idler) OPO output power from the non-planar RISTRA cavity. Right: The $\mathrm{M}^{2}$ time evolution measured with $20 \mathrm{~ms}$ resolution at maximum output power of $22 \mathrm{~W}$ from ZGP OPO in RISTRA cavity. The inset graph shows the example of a beam profile for the last point in the main graph.

For the first time we provided insight to how thermal effects in a non-linear crystal arise and evolve in time by measuring the $\mathrm{M}^{2}$ factor of the OPO beam as a time function. We investigated factors affecting the beam quality at high power levels like steady-state and transient thermal lens and methods to compensate for it. Our results can lead to better management of thermal effects and further up scaling of output power from ZGP OPO while maintaining good beam quality.

\section{References}

[1] S. Das, "Optical parametric oscillator: status of tunable radiation in mid-IR to IR spectral range based on ZnGeP2 crystal pumped by solid state lasers", Opt. Quantum Electron. 51, 70 (2019).

[2]. A. Hemming, et al., "99 W mid-IR operation of a ZGP OPO at 25\% duty cycle," Opt. Express 21, 10062-10069 (2013).

[3] C.-P. Qian et. al. "High repetition rate $102 \mathrm{~W}$ middle infrared ZnGeP2 master oscillator power amplifier system with thermal lens compensation," Opt. Lett. 44, 715-718 (2019).

[4] B.-R. Zhao, et. al. "231 W dual end-pumped Ho:YAG MOPA system and its application to a mid-infrared ZGP OPO," Opt. Lett. 43 5989-5992 (2018).

[5] M. Schellhorn and G. Spindler, "High-Efficient Resonantly Pumped Q-Switched Ho:LLF MOPA System", in Laser Congress 2019 (ASSL, LAC, LS\&C), OSA Technical Digest (Optical Society of America, 2019), paper JTh3A.18.

[6] A. V. Smith and M. S. Bowers, "Image-rotating cavity designs for improved beam quality in nanosecond optical parametric oscillators", J. Opt. Soc. Am. B 18, 706-713 (2001).

[7] S. Bigotta, G. Stöppler, J. Schöner, M. Schellhorn, and M. Eichhorn, "Novel non-planar ring cavity for enhanced beam quality in highpulse-energy optical parametric oscillators", Opt. Mater. Express 4, 411-423 (2014).

[8] M. Schellhorn, G. Spindler, and M. Eichhorn, "Improvement of the beam quality of a high-pulse-energy mid-infrared fractional-image-rotation-enhancement ZnGeP2 optical parametric oscillator", Opt. Lett. 42, 1185-1188 (2017). 